\title{
1 Bradykinin in Hemipepsis ustulata: a novel method for safely milking wasps
}

2 Shawn R. White ${ }^{\mathrm{a}}$ and Samuel Kadavakollu ${ }^{\mathrm{b}}$

3

4

5

6

7

8

9

10

11

12

13

14

15

16

17

18

19

20

21

22

23 Toxicon

24 Short Communication

25

26

27 a. Shawn R. White Ph.D.

Western New Mexico University

Department of Chemistry

1000 W. College Ave

Silver City, NM 88061 USA

Phone: (443)-523-2213

Email:whites7@wnmu.edu

b. Corresponding Author

Samuel Kadavakollu Ph.D.

Western New Mexico University

Department of Chemistry

1000 W. College Ave

Silver City, NM 88061 USA

Phone: (505)-803-4835

Email: samuelk@wnmu.edu 
ABSTRACT

Wasp venom characterization is of interest across multiple disciplines such as medicinal chemistry and evolutionary biology. A simple method is described herein to milk wasp venom without undue risks to the researcher. The wasps were immobilized by cooling for safe handling, restrained, and their venom was collected on parafilm. Bradykinin from Hemipepsis ustulata was identified by LC-MS/MS during method verification.

Keywords: Wasp; bradykinin; venom; milking; Hemipepsis ustulata; LC-MS/MS.

Wasp venoms have been affected by evolutionary processes based on their changing molecular targets and contain a variety of biologically active substances (Lee et al., 2016) that

help define metazoan taxa. In particular, solitary wasps contain neurotoxins such as bradykinins and pompilidotoxins that attack sodium channels, as well as a myriad of other diverse functioning compounds (Palma, 2006). Exploration of venom across families is important for biomedical research (lead drug candidates), although only a few species of solitary wasps have been studied (Konno et al., 2002; Baek et al., 2015). Methods of venom collection for social Hymenopterans (Benton et al.,1963) cannot be applied to solitary wasps. This short communication focuses on a novel milking technique suitable for large-sized, solitary wasps.

The method was tested on three individuals of Hemipepsis ustulata-large wasps (up to 5 $\mathrm{cm}$ ) with rust colored wings and iridescent blue bodies. Hemipepsis ustulata are ectoparasites that use paralyzed spiders as a food source for their young. These wasps are not aggressive, in general, but their sting has a fierce reputation (Schmidt, 2004) causing extreme, but short-lived pain. Although they are distributed worldwide, they are particularly well known in the southwest U.S., where they enjoy the status of being the state insect of New Mexico. 
The safest method for venom collection involves killing the insect and removing the

51 venom sac (Piek, 1986). This method has the disadvantage of producing a more complex sample

52 than that acquired from an external secretion. Three other methods in the literature describe

53 external venom collection, but all of these unnecessarily subject the researcher to the risk of

54 being stung. For instance, one method has the researcher using a piece of tape on their finger to

55 try to immobilize the active wasp by contact with its wings and then crushing the head with a pin

56 (Deyrup and Matthews, 2003). This method also carries the limitation of a one-time milking.

57 Another method suggests small wasps, such as jewel wasps (Ampulex compressa), can be milked

58 by forcing them stinger first into a narrow tube with a hole at the end and then confined with a

59 piston that serves also as a source of blunt trauma to induce venom secretion (Mukhopadhyay,

60 2014). Other researchers suggest picking up the wasp between the thumb and forefinger

61 (Sahayaraj et al., 2006). Most recently, an agar block-based method has been published

62 (Bhagavathula, et al., 2016), but collecting the venom in agar is unnecessary and introduces an

63 extra extraction step. The novel method described here uses a reduced-temperature incubation to

64 induce a state of immobilization to limit the exposure of the researcher to being stung. Parafilm

65 is used to receive the venom droplets.

Specimens were collected in Grant County, NM in the City of Rocks State Park and from

67 the campus of the Chiricahua Desert Museum in Rodeo, NM. The motor function of the wasps

68 was reduced by placing the cage in a refrigerator at $4{ }^{\circ} \mathrm{C}$. The wasp was checked on every 2

69 minutes until immobilized and then removed from the refrigerator. At this point, the wasp was

70 handled with tongs and its wings clipped (the wing venation was preserved for identification

71 purposes), an operation which facilitates ease of handling after the wasp reinvigorates. Narrow

72 strips of duct tape were used to secure the wasp in a supine position on a suitably-sized tube. The 
73 platform was securely taped down to the work surface to avoid the unexpected movement of the

74 stinger during milking. The wasp was ready for milking after 5-10 min.

75

76

77

78

79

80

81

82

83

84

At this point, a piece of parafilm $(4 \times 6 \mathrm{~cm})$ was held over the end of the abdomen to collect venom while the head of the wasp was pressed on without crushing to induce secretion (Fig.1). At this point, the stinger (up to $6 \mathrm{~mm}$ long) was easily observed and the parafilm was moved in contact with it. The stinger punctured the parafilm and deposited venom on the top side. The droplets were easily observed and were collected rapidly using a micropipetter and a small amount of water (5-10 $\mu \mathrm{L})$ to help dissolve the venom. After milking, the wasp was immobilized again using the refrigeration protocol. The tape was then carefully removed and the wasp returned to its cage. Cut grapes and wet paper towels were provided to sustain the wasps during captivity. This process was repeated for 2-4 days before the cumulative effect of the procedure became fatal.

The droplets secreted by Hemipepsis were around $1 \mu \mathrm{L}$ with about 4-8 droplets able to be collected in a single session. Venom samples were purified and preconcentrated with C-18 spin columns, lyophilized, and stored frozen until the time of analysis. Thawed venom was redissolved in $20 \mu \mathrm{L}$ of $0.1 \%$ formic acid solution and a trypsin digestion was performed to reduce coelution issues with proteins. A sample injection of $2 \mu \mathrm{L}$ was then analyzed by liquid chromatography-tandem mass spectrometry (LC-MS/MS) on a Q Exactive mass spectrometer with an Easy NanoLC-1000 system (Thermo Fisher Scientific, U.S.A). Separation was conducted using a C18 column (75 $\mu \mathrm{m}$ x $150 \mathrm{~mm})$. Reverse phase chromatography was performed with (A) $0.1 \%$ formic acid/water and (B) $0.1 \%$ formic acid/acetonitrile. A four-step, linear gradient was used for the LC separation (column pre-equilibration with 2\% B for 10 min; $2 \%$ to $30 \%$ B in the first 47 min.; followed by $80 \%$ B for 13 min.). Bradykinin was targeted in 
96 the data sets since bradykinin and related compounds have been found in other solitary wasp

97 species (Konno et al., 2002; Picolo et al., 2010).

These samples were concentrated enough to generate a large number of peaks in the main

99 chromatogram (Fig. 1). A mass range filter from 530.77-530.79 amu was applied to the total ion

100 chromatogram (Fig. 2a) to search for bradykinin (RPPGFSPFR), resulting in a strong peak at

101 retention time $15.6 \mathrm{~min}$. A match for the parent ion $\left(\mathrm{MH}_{2}{ }^{2+}\right)$ at monoisotopic mass $530.787 \mathrm{amu}$

102 was found (Fig. 2b) as the base peak in the mass spectrum (the online resource from University

103 of California San Francisco, Protein Prospector, was used to calculate the theoretical value of the

104 bradykinin peak at $530.788 \mathrm{amu}$ ). The doubly-charged state was deduced from the $0.5 \mathrm{amu}$

105 spacing of the envelope.

106 This paper presents a novel, effective (validated by LC-MS/MS) approach for milking

107 large wasps safely without the need for an agar block receiver. In addition, this method can

108 provide multiple samples from a single individual over time.

\section{Acknowledgements}

110 This work was supported by funding from the New Mexico Idea Networks for

111 Biomedical Research Excellence (NM-INBRE), NIH Prime Award No.: 2P20GM103451-14.

112 The authors would also like to thank Dr. Sucharita Dutta (Eastern Virginia Medical School), Dr.

113 Bill Toth and the Natural Sciences Department at WNMU. We are grateful to Justin Lewis,

114 James Sanders, and John Briggs for assistance in capturing wasp specimens and to Diana

115 Sanchez and Kay Lindsey for their assistance in milking wasps. 
116 Figure 1. Total ion chromatogram for Hemipepsis ustulata venom after trypsinolysis. Wasps 117 were restrained in a supine position and agitated to sting parafilm.

118

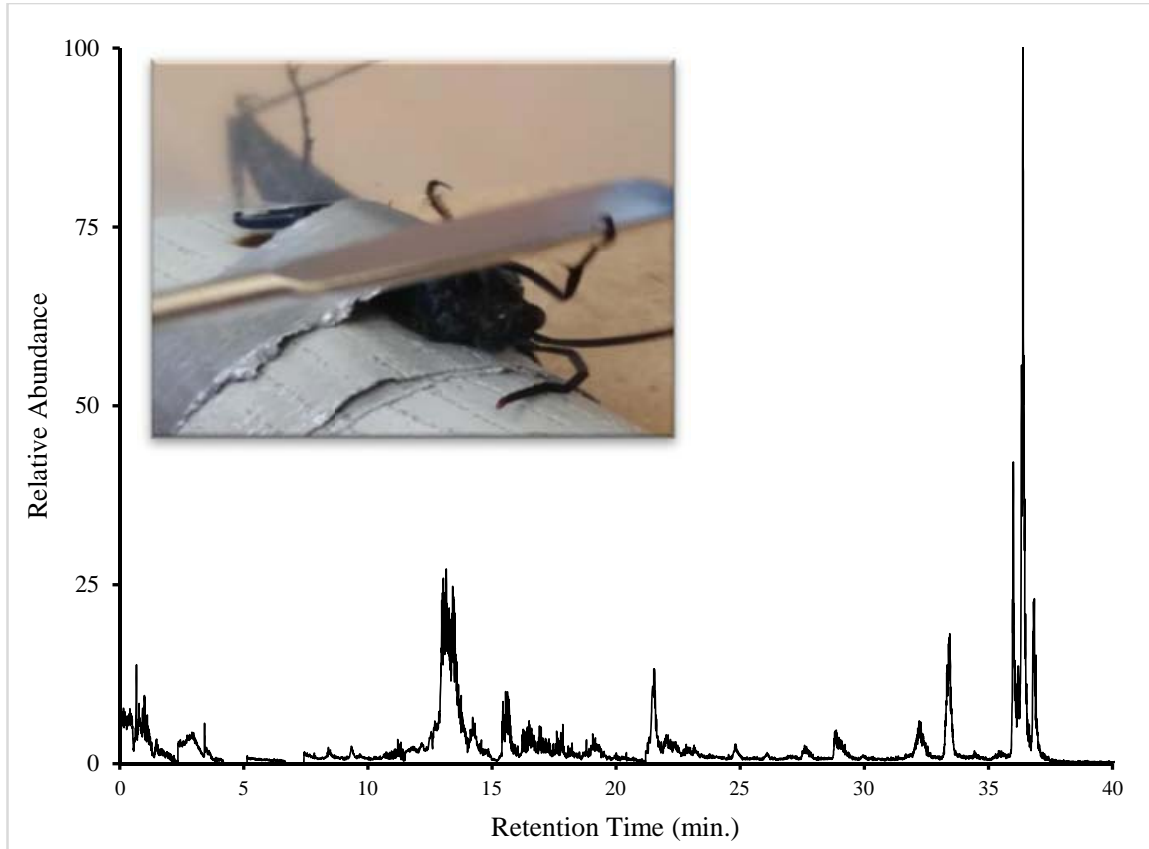

120 


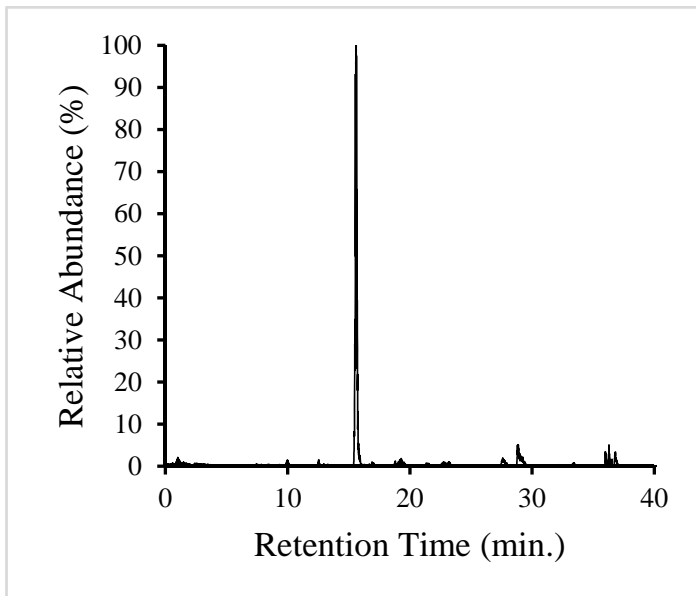

$1292 \mathrm{a}$

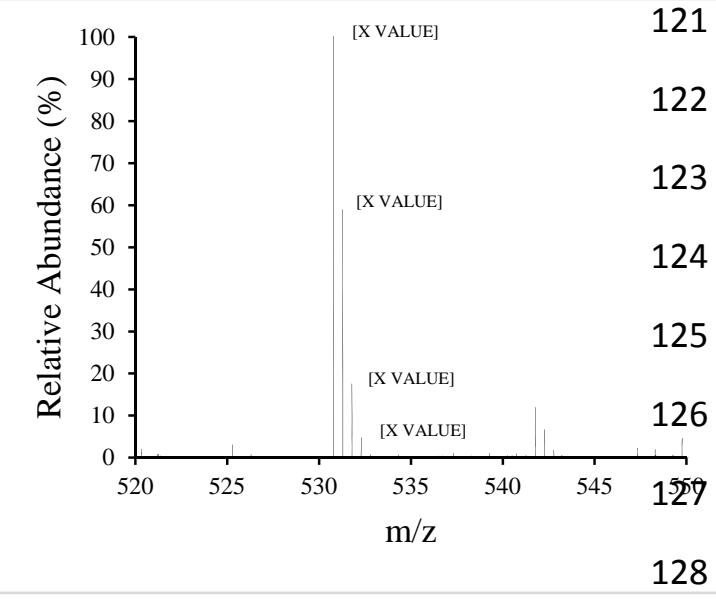

$2 \mathrm{~b}$

130 Figure 2a. Selected ion chromatogram for bradykinin at mass $530.79( \pm 0.5)$ amu.

131 Figure 2b. Mass spectrum for retention time $15.6 \mathrm{~min}$. (bradykinin) showing the isotopic 132 envelope for the doubly-charged, parent $\mathrm{MH}_{2}{ }^{2+}$ peak.

133

134 
Baek, J.H., Lee, S.H., Kim, W.Y., Kim, M.G., 2015. An insulin-binding protein from the venom of a solitary wasp Eumenes pomiformis binds to apolipophorin III in lepidopteran hemolymph. Toxicon 111, 62-64.

Benton, A.W., Morse, R.A., Stewart, J.D., 1963. Venom collection from honey bees. Science 142, 228230.

Bhagavathula, N.C., Kumar, M., Krishnappa, C., 2016. A simple non-invasive technique for venom milking from a solitary wasp Delta conoideum Gmelin (Hymenoptera: Vespidae). Toxicon 109, 4-6.

Deyrup, L.D., Matthews, R.W., 2003. A simple technique for milking the venom of a small parasitic wasp, Melittobia digitata (Hymenoptera: Eulophidae). Toxicon 42, 217-218.

Konno, K., Palma, M.S., Hitara, I.Y., Juliano, M.A., Juliano, L., Yasuhara, T., 2002. Identification of bradykinins in solitary wasp venoms. Toxicon 40, 309-312.

Lee, S.H., Baek, J.H., Yoon, K.A., 2016. Differential properties of venom peptides and proteins in solitary vs. social hunting wasps. Toxins 8, 1-29.

Mukhopadhyay, R., 2014. Under the spell of the cockroach hunter. ASBMB today 13, 32-39.

Palma, M.S., 2006. Insect Venom Peptides, in: Kastin, A.J. (Ed.), Handbook of Biologically Active Peptides, first ed. Elsevier, London, pp. 409-416.

Picolo, G., Hisada, M., Moura, A.B., Machado, M.F., Sciani, J.M., Conceicao, I.M., Melo, R.L., Oliveira, V., Lima-Landman, M.T., Cury, Y., Kono, K., Hayashi, M.A., 2010. Bradykinin-related peptides in the venom of the solitary wasp Cyphononyx fulvognathus. Biochem. Pharmacol. 79, 478-486.

Piek, T., 1986. Methods for the collection of venoms in: Piek, T. (Ed.), Venoms of the Hymenoptera, first ed. Academic Press, London, pp. 45-61.

Sahayaraj, K., Kumar, S.M., Arandh, G.P., 2006. Evaluation of milking and electric shock methods for venom collection from hunter reduviids. Entomon 31, 65-68.

Schmidt, J.O., 2004. Venom and the good life in tarantula hawks (Hymenoptera: Pompilidae): how to eat, not be eaten, and live long. J. Kansas Entomol. Soc. 77, 402-413. 